DR. ANJA LACHENMAYER (Orcid ID : 0000-0002-5879-5737)

Article type : Original Articles

Handling Associate Editor: Janus Ong

\title{
Stereotactic Image-Guided Microwave Ablation of Hepatocellular Carcinoma using a computer-assisted navigation system
}

Anja Lachenmayer ${ }^{*}$, Pascale Tinguely ${ }^{1}$, Martin Maurer $^{2}$, Lorenz Frehner ${ }^{1}$, Marina Knöpfli ${ }^{1}$, Matthias Peterhans ${ }^{3}$, Stefan Weber ${ }^{4}$, Jean-François Dufour ${ }^{1}$, Daniel Candinas ${ }^{1}$, Vanessa Banz ${ }^{1}$

\footnotetext{
${ }^{1}$ Department of Visceral Surgery and Medicine, Inselspital, Bern University Hospital, University of Bern, Bern, Switzerland.

2 Department of Radiology, Inselspital, Bern University Hospital and University of Bern, Bern, Switzerland.

${ }^{3}$ CAScination AG, Bern, Switzerland.

${ }^{4}$ ARTORG Center for Biomedical Engineering Research, University of Bern, Bern, Switzerland.
}

\section{*Corresponding author:}

Anja Lachenmayer, MD

Department of Visceral Surgery and Medicine

Inselspital, Bern University Hospital and University of Bern

CH-3010 Bern, Switzerland

Fax: $+4131 / 6329765$

Email: anja.lachenmayer@insel.ch

Telephone: +41316325948

This article has been accepted for publication and undergone full peer review but has not been through the copyediting, typesetting, pagination and proofreading process, which may lead to differences between this version and the Version of Record. Please cite this article as doi: $10.1111 /$ liv.14187

This article is protected by copyright. All rights reserved. 


\section{List of abbreviations in order of appearance:}

Hepatocellular carcinoma (HCC)

orthotopic liver transplantation (OLT)

Barcelona Clinic Liver Cancer classification (BCLC)

computed tomography (CT)

stereotactic image-guided microwave ablation (SMWA)

longitudinal positioning error (LPE)

magnetic resonance imaging (MRI)

hospital stay (LOS)

intrahepatic cholangiocarcinoma (ICC)

nonalcoholic steatohepatitis (NASH)

radiofrequency ablation (RFA)

\section{Conflict of interest declaration of all authors:}

Daniel Candinas, Stefan Weber and Matthias Peterhans hold shares of CAScination and Matthias Peterhans is employed by CAScination. All other authors have no conflicts of interest to declare.

\section{Statement of financial support:}

No funding of any of the authors was relevant to the study.

\section{Abstract}

Background \& Aims:

Ablation plays an important role in the treatment of hepatocellular carcinoma. Because imageguided navigation technology has recently entered the clinical setting, we aimed to analyze its safety, therapeutic and procedural efficiency.

Methods:

This article is protected by copyright. All rights reserved. 
Retrospective analysis of patients treated with stereotactic image-guided microwave ablation between $01 / 2015$ and 12/2017. Interventions were performed using computertomographyguidance with needle trajectory, ablation planning and automatic single-marker patient registration. Needle placement and ablation coverage was controlled by image fusion under general anesthesia with jet-ventilation.

Results:

In total 174 ablations were performed in 88 patients during 119 interventions. Mean age was 66 (46-84) years, 74 (84.1\%) were men and 74\% were Child Pugh Class A. Median tumor size was 16 (4-45) mm, 62.2\% were BCLC A. Median lateral and longitudinal error of needle placement were $3.2(0.2-14.1)$ and $1.6(0-15.8) \mathrm{mm}$. Median one tumor (1-4) was ablated per session. One patient developed a Dindo IIIb (0.8\%) complication, 6 minor complications. After re-ablation of 12 lesions, an efficacy rate of $96.3 \%$ was achieved. Local tumor progression was $6.3 \%(11 / 174)$. Close proximity to major vessels was significantly correlated with local tumor progression $(\mathrm{p}<0.05)$. Median overall follow-up was 17.5 months after intervention and 24 months after initial diagnosis. BCLC stage, child class and previous treatment were significantly correlated with overall survival $(\mathrm{p}<0.05)$.

\section{Conclusion:}

Stereotactic image-guided microwave ablation is a safe and efficient treatment for HCC offering a curative treatment approach in general and in particular for lesions not detectable on conventional imaging or untreatable due to difficult anatomic locations.

Key words: navigation, stereotactic, microwave ablation, hepatocellular carcinoma.

\section{Lay Summary / Key Points:}

Computer navigated minimal-invasive thermal ablation of hepatocellular carcinoma is a novel technique that allows a safe and efficient curative treatment in particular for inoperable, not detectable or conventionally unablatable liver lesions.

This article is protected by copyright. All rights reserved. 


\section{Introduction}

Hepatocellular carcinoma (HCC) is the second most common cause of cancer-related death and the main cause of death in patients with cirrhosis ${ }^{1}$. Curative treatment options for patients with early stage HCC include surgical resection, orthotopic liver transplantation (OLT) and local ablation 2. However, only a minority of patients qualify for a curative treatments due to advanced liver disease, intrahepatic tumor distribution or comorbidities ${ }^{3}$. Even potentially "ideal" transplant candidates may not go on to have the surgery they would actually require, with many transplant centers rarely adopting OLT as a first-line option for very early or early HCC ${ }^{4}$. Furthermore, as resection or ablation do not preclude salvage OLT in case of treatment failure, the use of initially less invasive strategies is often favored 5,6. Indeed, with 5-year overall survival rates of up to $75 \%$, ablation has been validated as an alternative to resection for very small tumors (Barcelona Clinic Liver Cancer classification (BCLC) stage 0) ${ }^{7}$ and patients with limited disease non-resectable due to associated liver disease (BCLC stage A) ${ }^{8}$. In addition, ablation has shown promising results within combination therapies for advanced disease 9,10 and is commonly used as bridging to OLT (as recommended by the current AASLD and EASL guidelines) ${ }^{11-13}$. The major advantage of ablation lies in its minimal-invasive tissue-sparing yet locally destructive character, with short hospital stays and low morbidity ${ }^{14}$. Although radiofrequency ablation (RFA) is used mostly for HCC, microwave ablation (MWA) has recently entered the clinical field and is now increasingly used due to shorter ablation times, a potential advantage for larger tumors and reduced heat sink effect, which occurs when hepatic blood flow reduces the local heat distribution by its cooling effect 15 .

Next to tumor burden and the extent of the underlying liver disease, initial complete response represents an independent predictive factor for recurrence-free and disease-specific survival in patients undergoing ablation for HCC ${ }^{16}$. Hence, the precision of ablation might represent a crucial factor for treatment success, relying directly on optimal visualization of intrahepatic lesions and accurate placement of ablation probes within the tumor targets. To optimize guidance of the ablation probe and thus precision in tumor targeting and ablation, advanced stereotactic navigation technology has been introduced, and several navigation systems are now available for image-guided interventions ${ }^{17-19}$. First reports on stereotactic percutaneous computed tomography (CT) - guided ablation of liver tumors have been published, suggesting a safe and accurate treatment ${ }^{20-22}$. While a few works report oncological benefits when using stereotactic ablation for colorectal liver metastases ${ }^{23}$, no data exists on oncologic outcomes after stereotactic CT-guided microwave ablation in a large cohort of HCC patients. Furthermore, difficult to detect lesions in ultrasound or CT and potentially inaccessible lesions are usually not taken into account in studies reporting HCC ablation results. Hence, understanding the clinical impact of using novel navigation technology for image-guided ablation of HCC remains scarce.

This article is protected by copyright. All rights reserved. 
Overall, we hypothesize that stereotactic image-guided microwave ablation (SMWA) is a safe and precise ablative treatment for all HCCs including those lesions that are conventionally ineligible to ablation (difficult anatomical location / not detectable on ultrasound or CT) or inoperable due to the underlying liver disease or patient comorbidities. In this first retrospective analysis, we report safety and therapeutic efficacy in terms of local tumor control and short-term survival, as well as procedural efficiency in terms of targeting accuracy and required time when using SMWA for the treatment of HCC.

\section{Materials and Methods}

All patients with HCC treated with SMWA at the Department of Visceral Surgery and Medicine and the Department of Radiology, University Hospital of Bern, Switzerland between January 2015 and December 2017 were retrospectively included in the analysis. The study protocol was approved by the Regional Ethical Review Board (KEK-No. 2017-01038), and registered at clinicaltrials.gov (NCT03630068).

\section{Patient population}

All patients were discussed in the institutional multidisciplinary tumor board. General indications for SMWA included patients with 1) single solitary lesions <2cm (BCLC 0), 2) single or up to 3 nodules $\leq 3 \mathrm{~cm}$ (BCLC A) not amenable to resection or OLT, 3) multinodular disease and/or tumors $>3 \mathrm{~cm}$ (BCLC B) whenever the tumor board agreed to perform a downstaging so that patients could be evaluated for liver transplantation ${ }^{24}$. Accordingly, SMWA was the method of choice as a bridge-to-transplant or as a downstaging procedure for patients awaiting OLT. SMWA is also the standard of care for all patients receiving an ablative treatment of HCC at our institution irrespective of the tumor location. Tumors had to be radiologically (using the LIRADS categorical system) 25 or histologically confirmed HCC requiring SMWA as indicated by the consensus tumor board decision. Written informed consent was obtained before the procedure in patients aged $\geq 18$ years.

\section{Procedural technique}

All procedures were performed under general anesthesia in the interventional CT suite by an interdisciplinary team consisting of specifically trained surgeons and interventional radiologists. Patients were placed on the CT table and High Frequency Jet ventilation was used to ensure minimal movement of the patient and the diaphragm during the procedure. The intervention always consisted of four main procedural steps: (1) planning, (2) navigation, (3)

This article is protected by copyright. All rights reserved. 
probe validation and ablation, (4) ablation zone validation (Figure 1). At first, a CT planning scan was performed (SOMATOM Definition Flash, Siemens Healthineers, Erlangen, Germany) using a predefined multi-phase imaging protocol $(2 \times 64 \times 0.6 \mathrm{~mm} ; 280 \mathrm{msec}$ gantry rotation time; pitch factor, 0.6; tube voltage; $100 \mathrm{kV}$ ) after the application of intravenous contrast medium (100-120 mL Ultravist ${ }^{\circledR}$ (volume depending on body mass of patients), Bayer Healthcare, Berlin, Germany) using bolus tracking (threshold $100 \mathrm{HU}$ ) for starting the scan: arterial phase 8 seconds after reaching the threshold, portal venous phase after another 25 seconds, and a late phase after another 150 seconds. The scan lengths was adapted to the size and height of the patients always including 6 fiducial markers attached to the patients' skin surface. The radiologist in the team reviewed all three CT phases to choose the scan in which the target lesion(s) was (were) best detectable. This scan was transferred to a commercially available navigation system (CAS-ONE, CAScination AG, Bern, Switzerland) which was then used for trajectory planning. After positioning of the ablation probe in the target lesion in the liver, a non-enhanced CT scan was performed to validate the correct positioning. The accuracy of needle placement with respect to the planned target point was evaluated by a fusion of the planning image with the needle control scan. The navigation system provides functionality for automatic image fusion, automatic needle detection and for calculation of lateral and longitudinal errors (distance between the active zone of the needle and the needle target point in the tumor. Thermal ablation of up to four lesions per patient was performed using microwave probes (Acculis MTA System, AngioDynamics, Latham, NY, USA). Finally, a contrast enhanced CT scan with three phases was performed in the same way as the planning scan to allow an immediate treatment validation of all target lesions by fusing both scans. The navigation system automatically performs a fusion between the planning and validation scan and allows for margin assessment by projecting the tumor segmentation from the planning image onto the validation scan. Further evaluation can be performed by image overlay where the planning and validation scans are displayed simultaneously and the user can switch back and forth between the images. This $50 / 50 \%$ display was frequently used to estimate if the planned safety margin was obtained. A safety margin of $0.5-1 \mathrm{~cm}$ was anticipated. If the validation showed an insufficient safety margin, a re-ablation was planned covering the area of the insufficient margin. The validation scan with the ablation zone could be fused with the pre-interventional scan to directly target the insufficiently ablated area.

\section{Data extraction and assessment}

Demographic and clinical data were extracted from patient's medical records. Lesion-specific and radiological data were extracted from DICOM imaging files, and technical data acquired during the intervention was extracted from the navigation system log files.

This article is protected by copyright. All rights reserved. 
To evaluate technical and procedural efficiency of SMWA, targeting accuracy assessed as lateral and longitudinal positioning error (LPE), time efforts required for the four procedural steps and overall duration of the procedure were measured. 90-day clinical and radiological complications were collected and graded according to the Dindo-Clavien classification ${ }^{26}$. The first follow-up imaging (magnetic resonance imaging (MRI) or CT) was carried out routinely 1-3 months post-treatment, with re-imaging every 3 months in case of stable disease. When MRI was performed, a specific liver imaging protocol was used including the hepatocyte-specific contrast medium gadoxetic acid (Primovist ${ }^{\mathrm{TM}} /$ Eovist $^{\mathrm{TM}}$, Bayer Healthcare Pharmaceuticals, Berlin, Germany). Technique efficacy was reported according to the standardized criteria suggested by Ahmed et al. 27, and 1) tumors showed no signs of residual tumor within $10 \mathrm{~mm}$ from the edge of the ablation zone on the first follow-up imaging (1-3 months postinterventional) or 2) tumors with residual tumor on the first follow-up scan were successfully re-ablated within 6 months. Local tumor progression was defined as the presence of detectable tumor within $10 \mathrm{~mm}$ from the edge of the ablation zone or the resection site on follow-up imaging after at least one prior follow-up imaging showed no residual tumor at the ablation zone/resection site. Disease progression was analyzed as either local tumor progression at the ablation zone, disease progression in the liver showing new liver lesions outside the ablated region and overall disease progression including distant metastases. Imaging results were interpreted and verified by two independent radiologists. The appearance of new intrahepatic or extrahepatic lesions was also documented. Transplantation was considered as a censored event for the survival analysis.

\section{Statistics}

Descriptive statistics were used for presentation of patient characteristics and outcome data. Continuous data are shown as mean and standard deviation or median and range where appropriate. The student T-Test was used to evaluate possible differences between groups. The Kaplan-Meier method, log rank test, logistic regression and cox regressions were applied to analyze the association of variables with local tumor progression, progression-free and overall survival. The threshold for statistical significance was set to $\mathrm{p} \leq 0.05$. Descriptive statistics and graphs were analyzed using SPSS Version 25.

This article is protected by copyright. All rights reserved. 


\section{Results}

\section{Clinical Data}

Over a period of 36 months, 88 , predominantly male patients were treated for 174 lesions in a total of 119 SMWA interventions at our institution (Table 1). Half of the patients $(n=44)$ had previously been treated for HCC by other means (Table 1). Sixteen (36.4\%) of these SMWA treated lesions were local tumor progressions after previous treatment failure, 28 (63.6\%) were new tumors located elsewhere in the liver. In 33 patients, SMWA was performed as a bridging procedure prior to liver transplantation.

Ninety-nine (83.2\%) interventions were performed in cirrhotic livers, 13 (10.9\%) in fibrotic liver, for seven (5.9\%) interventions the degree of underlying liver disease was unknown.

\section{HCC-specific data}

At the time of intervention the majority of patients were BCLC stage A (n=74,62.2\%) (Table 1). Treated lesions were located in all liver segments, including segment I (Figure 2). The majority of tumors were located in segment VIII (32.2\%), followed by a relatively even distribution of lesions in all other segments. While more than half of the treated lesions (59.2\%) were localized subcapsular (within 10mm), $29.9 \%$ were in close proximity (within $5 \mathrm{~mm}$ ) to major blood vessels (> 3mm). Among these, 32 lesions were located close to a portal vein branch, 11 close to a liver vein, one close to the vena cave and three close to the hepatic artery. Forty-five (25.9\%) lesions were within $10 \mathrm{~mm}$ of the diaphragm or heart. Eleven lesions were in close proximity to other organs including the gallbladder $(n=4)$, kidney $(n=2)$, duodenum $(n=1)$, stomach $(n=2)$ and colon (n=2) (Figure 2).

\section{Intervention-specific data}

The majority of patients had one $(n=77,64 \%)$ or two $(n=31,26.1 \%)$ tumors treated during one intervention. Nine patients had three (7.6\%) and two patients had four $(1.7 \%)$ lesions ablated during one intervention. All interventions were performed with microwave energy between 60 and 140 Watt for a median of five (1:30-18) minutes per tumor (Table 2). Thirteen lesions were treated with two needles and four lesions with three parallel needles to achieve overlapping ablation zones. We treated tumors $>3 \mathrm{~cm}$ significantly more often with multiple needles $(66.7 \%)$ than smaller lesions (33.3\%), ( $p>0.005$, Chi square test). For further technical details, see Table 2. The control of the ablation zone revealed an insufficient margin for 26 lesions immediately post-ablation, 19 of which could be immediately re-ablated. Three lesions could not be re-treated due to close proximity of the region of a potential insufficient margin to a major blood vessel $(n=1)$, gallbladder $(n=1)$, or lung $(n=1)$. One lesion was ablated to prepare a

This article is protected by copyright. All rights reserved. 
contralateral hepatectomy and three lesions were planned for an early follow-up. For 13 interventions, post-interventional imaging showed small intrahepatic or subcapsular hematomas and after one procedure a small pneumothorax was detected. None of these radiological findings were clinically symptomatic and they did not require any further treatment or follow-up. These incidental findings due to the detailed post-ablation scan were therefore not considered complications.

\section{Post-interventional Data}

Median length of hospital stay (LOS) after the intervention was two (range 1 - 4) days. The overall 90-day complication rate was 5.9\% (7/119), with six grade I-IIIa and one grade IIIb complication (Table 3). Grade I-IIIa complications included two ascites decompensations, one pleural effusion and one pneumothorax requiring drainage, one patient with postinterventional pain exacerbation and one patient with post-interventional fever treated with antibiotics. The patient with the Dindo grade IIIb complication required surgery for a suspected abscess (diagnosed on radiological imaging and fever), which was proven neither histologically nor in microbiology tests and probably represented a case of post ablation syndrome with fever. While we did not observe significant changes in the hemoglobin and creatinine levels before and after the intervention, the alanine transaminase increased significantly from a median 38 $\mathrm{U} / \mathrm{l}$ to $90 \mathrm{U} / \mathrm{l}(\mathrm{p}=0.000)$ without any clinical correlation. This increase was not considered a pathological finding as it reflects the destruction of liver tissue through ablation.

\section{Local tumor control and short-term survival}

The overall rate of residual tumor per lesion after the first intervention was $12.1 \%(21 / 174)$ at a 1-3 months follow-up. Of these, 12 (6.9\%) were successfully re-ablated in a second intervention within 6 months of the initial treatment, resulting in an efficacy rate of $96.3 \%$ (165/174). Nine lesions (5.2\%) with residual tumor at 1-3 follow-up received no additional ablation for varying reasons: two lesions turned out to be an intrahepatic cholangiocarcinoma (ICC) (among other HCC lesions in a nonalcoholic steatohepatits (NASH) liver) and a mixed HCC/ICC (among other HCC lesions), respectively, and treatment was switched to systemic therapy. Two patients were planned for re-ablation but were transplanted just prior to receiving their SMWA, two patients were treated by laparoscopic ablation and resection, respectively, two patients had progressive disease and treatment was switched to systemic therapy, one patient was lost to follow-up. Eleven lesions (6.3\%) developed a local tumor progression after 6 months of follow-up and at least one local tumor progression-free imaging. While nine $(5.7 \%)$ of these lesions were successfully re-ablated, one patient was transplanted

This article is protected by copyright. All rights reserved. 
before a re-ablation could be performed and one patient showed additional new intrahepatic lesions requiring a change to systemic therapy.

Close proximity to a major blood vessel $(\mathrm{p}=0.014)$ was the only factor significantly correlated with local tumor progression in the logistic regression analysis (Table 4). Tumor size $\geq 3 \mathrm{~cm}$ showed a trend to an inverse correlation with recurrence.

In the time to local tumor progression analysis (Figure 3A) tumor size $>3 \mathrm{~cm}$ (log rank test $\mathrm{p}=0.015$ 95\% CI: 2.359-18.641) was significantly correlated with local tumor progression-free survival.

Overall median follow-up from the intervention to death or end of follow-up was 17.25 (1.448.1) months, and 24 (1.4-127.8) months from initial diagnosis to death or end of follow-up. Follow-up ended at the time of transplantation. Twenty-five $(28.4 \%)$ patients died during this follow-up period, 17 (19.3\%) due to tumor progression (14 with HCC, one patient developed an acute myeloid leucemia, one patient a renal cell cancer and one patient developed an intrahepatic cholangiocarcinoma). Four patients died due to decompensated cirrhosis, three due to septic complications not related to the intervention and for one patient, cause of death remained unknown.

BCLC stage, child class and previous treatment within the last 2 years before SMWA were significantly correlated with survival /drop-out due to OLT in the univariate analysis (Figure 3B-D) and remained significant in the multivariate analysis (Supplementary Table 1).

\section{SMWA as bridging therapy}

Thirty-three (37.5\%) patients awaiting transplantation underwent SMWA as a bridging procedure. Out of these patients, 22 were transplanted after a median time of 10.7 (1.1 - 25.4) months, having undergone a median of one (1 - 4) SMWA treatment. Thirteen (65\%) had combined bridging treatments (five SMWA and TA(C)E, 6 SMWA and resection, 2 SMWA and laparoscopic ablation). Eleven patients dropped out of the waiting list. This included disease progression outside Milan criteria $(n=6)$, development of other cancers $(n=3)$ and other reasons $(\mathrm{n}=2)$. All transplanted patients remain alive to date after a median follow-up of $7.1(0-24.2)$ months post-transplant. Analysis of 40 ablation zones in the 22 explanted livers showed complete necrosis in $26 / 40$ (65\%) treated lesions with a mean rate of necrosis of $87 \%(15-100)$ (Figure 4).

This article is protected by copyright. All rights reserved. 


\section{Discussion}

In this large retrospective analysis, we report our experience with stereotactic-image-guided microwave ablation of HCC and show that SMWA is a safe and efficient minimally invasive treatment option achieving good oncological outcome and local tumor control.

Current guidelines still recommend RFA as the standard of care for the percutaneous treatment of HCC based on good local tumor control, with local recurrence rates between 3.2 and $28.5 \%$ at 3 years and an overall survival between 60 and $84.1 \%$ at 3 years ${ }^{28}$. This relatively large variation in local recurrence rates might be explained by the use of different technical devices, differences in image-guidance and also by not applying a uniform terminology for what is meant by local recurrence in particular with respect to the development of new intrahepatic lesions. A possible large variation of technical success rates among different centers might also be the reason, which is why a recent publication stated that ablation is correlated with worse overall survival compared with resection ${ }^{29}$.

More recently, RFA was challenged by MWA, which allows for faster treatment of larger tumors with greatly reduced heat-sink effect, a process that describes a decrease in local efficacy due to the cooling effect of blood flow in major vessels in proximity of the lesion to be ablated 30 . However, data on large patient series comparing both approaches are still rare. Nevertheless, MWA is increasingly being used for the percutaneous treatment of HCC with reported local tumor progression rates between $3.9-17 \%$ and an overall survival between 49 and $86.1 \%$ 28. A recent meta-analysis by Luo et al. demonstrated similar rates of local tumor control and overall survival when MWA and RFA were compared, with a slight benefit for MWA for the treatment of tumors larger than $3 \mathrm{~cm}^{31}$.

The treatment algorithm for HCC is highly complex and even though the BCLC classification is widely accepted, centers often decide differently for their patients in the "real life" setting. This is partly due to differences in organ availability and allocation, but mostly due to the complicated interplay of the degree of the underlying liver disease, the extent of relevant comorbidities, the anatomical location of the tumor and the on-site clinical / interventional expertise and the availability of modern technology allowing minimally invasive treatment approaches. All this makes the design of prospective trials almost impossible and explains the tremendous selection bias in most of the retrospective analyses in this field. Several studies suggest that up to a third of patients with early HCC end up receiving palliative therapies, rather than undergoing a curative approach as recommended for patients with early disease stages 32 , 33. This might in part be attributed to tumor location, such that patients with "difficult to reach" lesions or those not detectable on ultrasound or CT end up being placed in the palliative

This article is protected by copyright. All rights reserved. 
treatment group instead. In addition, most transplant candidates with BCLC A will not receive an up-front transplantation and thus need to be bridged with the most effective local treatments available.

Even though ablation may be considered as a minimally invasive procedure in itself, its feasibility is highly dependent on tumor visibility and the exact anatomical location of the lesion within the liver. It is known that up to a third of lesions remain "unablatable" in the clinical setting due to tumor location (proximity to critical structures), failure to detect the lesion on ultrasound or CT and high risk patients unsuitable for the intervention ${ }^{34}$. With this in mind, navigation technology has entered the field of hepatobiliary surgery and interventional radiology, allowing pre-interventional planning, precise navigated targeting and validation for immediate control of the treatment success. In our patient series, all patients suggested for microwave ablation therapy as agreed upon in our multidisciplinary tumor board, received SMWA, even if some lesions might have been ablatable by the conventional free-hand and ultrasound-guided approach (see also figure 2 for details). Indeed, the majority of our patients had tumors located in difficult to treat areas (close proximity to large vessels and/or bile ducts, sub-diaphragmatic lesions or subcapsular locations close to adjacent organs) and did not need additional supportive techniques such as hydro dissection using artificial ascites to overcome anatomical problems and to place the ablation probe ${ }^{35,36}$. Our high precision of needle placement is further reflected by the low mean lateral and longitudinal error of 3.2 and $1.6 \mathrm{~mm}$ and the fact that almost no needle replacements were necessary in our cohort. Few cases with relatively high lateral and longitudinal errors received no replacement of the ablation probe because the corrected simulated ablation zone for the deviated probe was still sufficiently covering the lesion or because the interventionalist preferred to ablate any resulting insufficient margin after performing the validation scan. The geometrical accuracy of $3.2 \mathrm{~mm}$ median lateral error achieved with CAS-One IR navigation system is comparable to similar navigation approaches in the literature. The group in Innsbruck reports lateral errors of $3.6 \pm 2.5 \mathrm{~mm}$ in a series of 145 needle placements on 20 patients using an optical navigation system ${ }^{37}$. Users of a robotic navigation system report applicator active point deviation after the first needle insertion of $5.3 \pm 1.8 \mathrm{~mm}^{38}$ and $3.1 \pm 2.5 \mathrm{~mm}{ }^{39}$ for series of 21 resp. 34 patients. In both reports, manual repositioning of the needle was performed and led to final positioning errors of $1.9 \pm 1.7$ $\mathrm{mm}$ resp. $1.6 \pm 1.3 \mathrm{~mm}$. A randomized study on computer assisted electromagnetic navigation shows a median initial targeting accuracy of $4.1 \mathrm{~mm}$ for 60 needle placements ${ }^{40}$. In this study too, further needle corrections where performed manually after the initial navigated needle placement. Comparing these results, we see that our results of $3.2 \mathrm{~mm}$ lie within the range of 3.1 to $5.3 \mathrm{~mm}$ reported in literature. Given, the high unpredictability of ablation zones with a given power/time setting ${ }^{41}$, we see the ablation validation using a post-ablation control scan as a

This article is protected by copyright. All rights reserved. 
crucial step for obtaining reliable tumor control in one session. In our treatment algorithm, the ablation prediction (based on manufacturer's datasheets) is used as an estimate for the required ablation energy. Independent of this prediction, we always use the navigation software to fuse pre- and post-ablation images in order to reliably validate the achieved ablation zone and trigger a re-ablation in the same session when insufficient margins are observed. A further aspect in standardizing ablation treatment is the use of JET-ventilation which guarantees minimal breathing movements that are required to achieve the precise placement of probes. Overall, our highly standardized protocol with reproducible workflows probably contributes to our low intra- and post-interventional complication rates. Although many argue that using general anesthesia and computer navigation for liver ablation leads to prolonged intervention times, there is not much data supporting this assumption. Indeed, Beyer et al. showed that procedure times were not increased when using SMWA for liver tumor ablation ${ }^{42}$. With the help of navigation technology, all patients in our cohort successfully underwent SMWA, even though in some instances a fusion with the pre-interventional MRI image had to be performed to visualize the lesions. So-called "invisible" lesions or lesions in the sub centimeter range were therefore no hindrance for treatment, a problem that is commonly described in US- (and CT-) guided ablations. Tumor seeding could be avoided in all cases due to the exact planning of the needle insertion site and needle tract ablation.

Although it has been widely published that complication rates after local ablation are significantly reduced compared to local resection ${ }^{43,44}$, data is limited on patients treated with SMWA. Our patient series shows a very low complication rate compared to most of the literature, accompanied by a short hospital stay with quick recovery. Engstrand et al. using the same navigation system for liver ablations also recently reported similar results 21 .

Even though the focus of our retrospective analysis was safety, and local efficacy of SMWA, short-term survival analyses showed that BCLC stage B, Child class and previous HCC treatment were significantly correlated with poor outcome. Our cohort reflects the fact that patients often receive multiple different and personalized treatments in the course of the disease hampering clear survival analyses of ablated HCC lesions. As previously reported, tumor size was significantly correlated with the development of local tumor progression. This might simply be due to the limitations of tissue destruction by microwave energy and future studies clearly need to show if new multi-probe ablation systems creating overlapping ablation zones will help to overcome this problem ${ }^{22}$. While we were able to see worse local tumor-progression-free survival in patients treated for HCCs $>3 \mathrm{~cm}$, we could not detect tumor size as a predictor of local tumor progression. In our cohort, most tumors with this size were treated with multiple parallel needles, eventually explaining our observation of even a trend towards an inverse correlation.

This article is protected by copyright. All rights reserved. 
In our view, the underlying degree of liver disease with its varying degree of liver stiffness might influence the size of the ablation zone and ultimately the treatment success. More detailed analyses of the underlying liver disease might help to develop predictors for local tumor progression and recurrence and to accurately plan ablation power and time with respect to the degree of steatosis, fibrosis or cirrhosis. Due to the retrospective nature of this study, the local tumor control rate and the overall survival rate could not be compared to another conventional treatment in our cohort. According to our treatment algorithm, SMWA is recommended for all BCLC 0 and A patients and for unresectable BCLC B patients either as a downstaging before OLT or in a curative intent whenever overlapping ablations zones can achieve a technical success with sufficient margins.

Many ( $>37 \%$ ) of our patients received SMWA as a bridging therapy to transplant. With average waiting times in Switzerland currently being around 12 months (www.swisstransplant.org), our aim was to use a potentially curative approach, with as many candidates as possible achieving complete pathological response (as documented in the explant) and as low as possible waiting list drop-out rates due to tumor progression. While our retrospective analysis of the explant's H\&E histology report only used the estimation of the pathologist (mean rate of necrosis 87\%), a recent paper by Bale et al. showed a complete pathological response of $97.3 \%$ when explants were analyzed by H\&E and an apoptosis assay, because it had been shown earlier that conventional H\&E staining underestimates the degree of necrosis ${ }^{45}$. With this in mind, we assume to achieve similar success rates with our technique.

Cancer treatment has changed dramatically over the last few years, with "one therapy fits all" regimes being replaced by patient-tailored, individualized strategies for a variety of cancers including HCC. Hereby, interventions, treatments, or other medical decisions are specifically designed for the individual patient, based on their predicted response or risk of disease ${ }^{46}$. Increasingly, patients with advanced age and concomitant comorbidities but limited disease are also being put forward for oncological treatments with curative intent. This patient population particularly profits from locally effective, but low risk, tissue-sparing interventions, where repeat therapy sessions are well tolerated even in case of local tumor progression $[10,11]$. In conclusion, SMWA using a computer-assisted navigation system is a safe and locally effective procedure for patients with HCC. While it improves the eligibility to a potentially curative and minimally invasive treatment option for patients conventionally unablatable it also represents a promising treatment alternative for lesions unresectable due to patient and liver comorbidity. Highly standardized treatment protocols as well as a specifically dedicated team, put together of hepatobiliary surgeons as well as interventional radiologists, ensure procedural safety while maximizing oncological outcome.

This article is protected by copyright. All rights reserved. 


\section{References}

1. Ferlay J, Soerjomataram I, Dikshit R, et al. Cancer incidence and mortality worldwide: sources, methods and major patterns in GLOBOCAN 2012. Int J Cancer 2015; 136(5): E359-86.

2. Bruix J, Reig M, Sherman M. Evidence-based Diagnosis, Staging, and Treatment of Patients With Hepatocellular Carcinoma. Gastroenterology 2016.

3. Bruix J, Sherman M, American Association for the Study of Liver D. Management of hepatocellular carcinoma: an update. Hepatology 2011; 53(3): 1020-2.

4. Scatton O, Goumard C, Cauchy F, et al. Early and resectable HCC: Definition and validation of a subgroup of patients who could avoid liver transplantation. J Surg Oncol 2015; 111(8): 1007-15.

5. Torzilli G, Belghiti J, Kokudo N, et al. A snapshot of the effective indications and results of surgery for hepatocellular carcinoma in tertiary referral centers: is it adherent to the EASL/AASLD recommendations?: an observational study of the HCC East-West study group. Ann Surg 2013; 257(5): 929-37.

6. Spolverato G, Vitale A, Ejaz A, et al. The relative net health benefit of liver resection, ablation, and transplantation for early hepatocellular carcinoma. World journal of surgery 2015; 39(6): 1474-84.

7. Chen M S, Li J Q, Zheng Y, et al. A prospective randomized trial comparing percutaneous local ablative therapy and partial hepatectomy for small hepatocellular carcinoma. Ann Surg 2006; 243(3): 321-8.

8. De Jong K P, Wertenbroek M W. Liver resection combined with local ablation: where are the limits? Digestive surgery 2011; 28(2): 127-33.

9. Katsanos K, Kitrou P, Spiliopoulos S, Maroulis I, Petsas T, Karnabatidis D. Comparative effectiveness of different transarterial embolization therapies alone or in combination with local ablative or adjuvant systemic treatments for unresectable hepatocellular carcinoma: A network meta-analysis of randomized controlled trials. PLoS One 2017; 12(9): e0184597.

10. Wang W, Shi J, Xie W F. Transarterial chemoembolization in combination with percutaneous ablation therapy in unresectable hepatocellular carcinoma: a meta-analysis. Liver international : official journal of the International Association for the Study of the Liver 2010; 30(5): 741-9.

11. Kulik L, Heimbach J K, Zaiem F, et al. Therapies for patients with hepatocellular carcinoma awaiting liver transplantation: A systematic review and meta-analysis. Hepatology 2018; 67(1): 381-400.

12. European Association for the Study of the Liver. Electronic Address E E E, European Association for the Study of The L. EASL Clinical Practice Guidelines: Management of

This article is protected by copyright. All rights reserved. 
hepatocellular carcinoma. J Hepatol 2018; 69(1): 182-236.

13. Heimbach J K, Kulik L M, Finn R S, et al. AASLD guidelines for the treatment of hepatocellular carcinoma. Hepatology 2018; 67(1): 358-80.

14. Ng K K C, Chok K S H, Chan A C Y, et al. Randomized clinical trial of hepatic resection versus radiofrequency ablation for early-stage hepatocellular carcinoma. The British journal of surgery 2017; 104(13): 1775-84.

15. Lencioni R, De Baere T, Martin R C, Nutting C W, Narayanan G. Image-Guided Ablation of Malignant Liver Tumors: Recommendations for Clinical Validation of Novel Thermal and NonThermal Technologies - A Western Perspective. Liver Cancer 2015; 4(4): 208-14.

16. Agopian V G, Harlander-Locke M P, Ruiz R M, et al. Impact of Pretransplant Bridging Locoregional Therapy for Patients With Hepatocellular Carcinoma Within Milan Criteria Undergoing Liver Transplantation: Analysis of 3601 Patients From the US Multicenter HCC Transplant Consortium. Ann Surg 2017; 266(3): 525-35.

17. Tinguely P, Fusaglia M, Freedman J, et al. Laparoscopic image-based navigation for microwave ablation of liver tumors-A multi-center study. Surgical endoscopy 2017; 31(10): 4315-24.

18. Banz V M, Muller P C, Tinguely P, et al. Intraoperative image-guided navigation system: development and applicability in 65 patients undergoing liver surgery. Langenbeck's archives of surgery 2016; 401(4): 495-502.

19. Fukuda H, Numata K, Nozaki A, et al. Usefulness of US-CT 3D dual imaging for the planning and monitoring of hepatocellular carcinoma treatment using HIFU. European journal of radiology 2011; 80(3): e306-10.

20. Widmann G, Schullian P, Haidu M, Bale R. Stereotactic radiofrequency ablation (SRFA) of liver lesions: technique effectiveness, safety, and interoperator performance. Cardiovascular and interventional radiology 2012; 35(3): 570-80.

21. Engstrand J, Toporek G, Harbut P, Jonas E, Nilsson H, Freedman J. Stereotactic CT-Guided Percutaneous Microwave Ablation of Liver Tumors With the Use of High-Frequency Jet Ventilation: An Accuracy and Procedural Safety Study. AJR American journal of roentgenology 2017; 208(1): 193-200.

22. Bale R, Schullian P, Eberle G, et al. Stereotactic radiofrequency ablation of hepatocellular carcinoma - A histopathological study in explanted livers. Hepatology 2018.

23. Engstrand J, Nilsson $\mathrm{H}$, Jansson A, et al. A multiple microwave ablation strategy in patients with initially unresectable colorectal cancer liver metastases - A safety and feasibility study of a new concept. European journal of surgical oncology : the journal of the European Society of Surgical Oncology and the British Association of Surgical Oncology 2014; 40(11): 1488-93.

This article is protected by copyright. All rights reserved. 
24. Forner A, Reig M, Bruix J. Hepatocellular carcinoma. Lancet 2018; 391(10127): 1301-14. 25. Elsayes K M, Hooker J C, Agrons M M, et al. 2017 Version of LI-RADS for CT and MR Imaging: An Update. Radiographics : a review publication of the Radiological Society of North America, Inc 2017; 37(7): 1994-2017.

26. Dindo D, Demartines N, Clavien P A. Classification of surgical complications: a new proposal with evaluation in a cohort of 6336 patients and results of a survey. Ann Surg 2004; 240(2): 205-13.

27. Ahmed M, Technology Assessment Committee of the Society of Interventional R. Imageguided tumor ablation: standardization of terminology and reporting criteria--a 10-year update: supplement to the consensus document. Journal of vascular and interventional radiology : JVIR 2014; 25(11): 1706-8.

28. Nault J C, Sutter O, Nahon P, Ganne-Carrie N, Seror O. Percutaneous treatment of hepatocellular carcinoma: State of the art and innovations. J Hepatol 2017.

29. Kolarich A R, Cabrera R, Hughes S J, George T J, Jr., Geller B S, Grajo J R. Thermal ablation versus wedge or segmental resection in patients with early stage hepatocellular carcinoma: a population survival analysis. HPB : the official journal of the International Hepato Pancreato Biliary Association 2019; 21(2): 249-57.

30. Kang $\mathrm{T} \mathrm{W}$, Lim $\mathrm{H} \mathrm{K}$, Lee $\mathrm{M} \mathrm{W}$, et al. Aggressive Intrasegmental Recurrence of Hepatocellular Carcinoma after Radiofrequency Ablation: Risk Factors and Clinical Significance. Radiology 2015; 276(1): 274-85.

31. Luo W, Zhang $\mathrm{Y}, \mathrm{He} \mathrm{G}$, et al. Effects of radiofrequency ablation versus other ablating techniques on hepatocellular carcinomas: a systematic review and meta-analysis. World journal of surgical oncology 2017; 15(1): 126.

32. Charriere B, Muscari F, Maulat C, et al. Outcomes of patients with hepatocellular carcinoma are determined in multidisciplinary team meetings. J Surg Oncol 2017; 115(3): 33036.

33. Leoni S, Piscaglia F, Serio I, et al. Adherence to AASLD guidelines for the treatment of hepatocellular carcinoma in clinical practice: experience of the Bologna Liver Oncology Group. Digestive and liver disease : official journal of the Italian Society of Gastroenterology and the Italian Association for the Study of the Liver 2014; 46(6): 549-55.

34. Kim Y S, Lim H K, Rhim H, et al. Ten-year outcomes of percutaneous radiofrequency ablation as first-line therapy of early hepatocellular carcinoma: analysis of prognostic factors. J Hepatol 2013; 58(1): 89-97.

35. Kang $\mathrm{T} \mathrm{W}$, Lim $\mathrm{H}$ K, Lee $\mathrm{M}$ W, Kim $\mathrm{Y} \mathrm{S}$, Choi D, Rhim H. Perivascular versus nonperivascular small HCC treated with percutaneous RF ablation: retrospective comparison of long-term therapeutic outcomes. Radiology 2014; 270(3): 888-99.

This article is protected by copyright. All rights reserved. 
36. Teratani T, Yoshida H, Shiina S, et al. Radiofrequency ablation for hepatocellular carcinoma in so-called high-risk locations. Hepatology 2006; 43(5): 1101-8.

37. Widmann G, Schullian P, Haidu M, Fasser M, Bale R. Targeting accuracy of CT-guided stereotaxy for radiofrequency ablation of liver tumours. Minimally invasive therapy \& allied technologies : MITAT : official journal of the Society for Minimally Invasive Therapy 2011; 20(4): 218-25.

38. Mbalisike E C, Vogl T J, Zangos S, Eichler K, Balakrishnan P, Paul J. Image-guided microwave thermoablation of hepatic tumours using novel robotic guidance: an early experience. Eur Radiol 2015; 25(2): 454-62.

39. Beyer L P, Pregler B, Niessen C, et al. Robot-assisted microwave thermoablation of liver tumors: a single-center experience. International journal of computer assisted radiology and surgery 2016; 11(2): 253-9.

40. Durand P, Moreau-Gaudry A, Silvent A S, et al. Computer assisted electromagnetic navigation improves accuracy in computed tomography guided interventions: A prospective randomized clinical trial. PLoS One 2017; 12(3): e0173751.

41. Ruiter S J S, Heerink W J, De Jong K P. Liver microwave ablation: a systematic review of various FDA-approved systems. Eur Radiol 2018.

42. Beyer L P, Lurken L, Verloh N, et al. Stereotactically navigated percutaneous microwave ablation (MWA) compared to conventional MWA: a matched pair analysis. International journal of computer assisted radiology and surgery 2018.

43. Feng K, Yan J, Li X, et al. A randomized controlled trial of radiofrequency ablation and surgical resection in the treatment of small hepatocellular carcinoma. J Hepatol 2012; 57(4): 794-802.

44. Huang J, Yan L, Cheng Z, et al. A randomized trial comparing radiofrequency ablation and surgical resection for HCC conforming to the Milan criteria. Ann Surg 2010; 252(6): 903-12. 45. Bale R, Schullian P, Eberle G, et al. Stereotactic Radiofrequency Ablation of Hepatocellular Carcinoma: a Histopathological Study in Explanted Livers. Hepatology 2018.

46. Moscow J A, Fojo T, Schilsky R L. The evidence framework for precision cancer medicine. Nat Rev Clin Oncol 2018; 15(3): 183-92.

This article is protected by copyright. All rights reserved. 


\section{Tables}

Table 1. Clinical Data

\begin{tabular}{|c|c|}
\hline Number of patients & 88 \\
\hline Number of interventions & 119 \\
\hline Number of ablated lesions & 174 \\
\hline Gender male & $74 / 88(84.1 \%)$ \\
\hline Median (range) age at intervention & $66(46-84)$ \\
\hline Number of patients previously treated for HCC & $44(50 \%)$ \\
\hline Resection & $15(17 \%)$ \\
\hline TAE/TACE & $17(19.3 \%)$ \\
\hline Ultrasound-guided Ablation & $12(13.6 \%)$ \\
\hline Number of patients awaiting transplantation & $33(37.5 \%)$ \\
\hline \multicolumn{2}{|l|}{ Child-Pugh stage at intervention } \\
\hline Child A & $88 / 119(74 \%)$ \\
\hline Child B & $31 / 119(26 \%)$ \\
\hline \multicolumn{2}{|l|}{ Etiology of liver disease } \\
\hline $\mathrm{HCV}$ & $32(36.4 \%)$ \\
\hline HBV & $4(4.5 \%)$ \\
\hline $\mathrm{HCV}+\mathrm{HBV}$ & $2(2.3 \%)$ \\
\hline Alcohol & $18(20.5 \%)$ \\
\hline NASH & $10(11.4 \%)$ \\
\hline ASH/NASH & $10(11.4 \%)$ \\
\hline Other* & $12(13.6 \%)$ \\
\hline \multicolumn{2}{|l|}{ BCLC stage at intervention } \\
\hline BCLC 0 & $39(32.8 \%)$ \\
\hline BCLC A & $74(62.2 \%)$ \\
\hline BCLC B & $6(5 \%)$ \\
\hline Median tumor size at intervention & $16(4-45) \mathrm{mm}$ \\
\hline Median AFP level at intervention & $5.6(1.6-3241) \mathrm{kU} / 1$ \\
\hline
\end{tabular}

This article is protected by copyright. All rights reserved. 


\section{Ablation}

Median ablation power

Geometric Accuracy

Median lateral error

Median longitudinal error

Time

Median time planning

Median time navigation time

Median ablation time

Median time validation ablation

Overall intervention

\section{Imaging}

Median amount of contrast agent (ml)

Median dose DLP $\left(\mathrm{mGy}^{*} \mathrm{~cm}\right)$
100 Watt $(60-140)$

$3.2 \mathrm{~mm}(0.2-14.1)$

$1.6 \mathrm{~mm}(0-15.8)$

$10.5 \min (1.3-55.6)$

$7.1 \mathrm{~min}(0.6-25.2)$

$5 \min (1.5-18)$

$3.8 \min (0.1-19.6)$

$36(3.6-88)$

$200(90-300)$

$1896(571-4748)$

Table 3. Complications

$\begin{array}{cl}\text { Peri-interventional } & 0(0 \%) \\ \text { Post-interventional } & 7(5.9 \%) \\ \text { Dindo grade I-IIIa } & 6(5 \%) \\ \text { Ascites decompensation } & 2 \\ \text { Pleural effusion } & 1 \\ \text { Pneumothorax } & 1 \\ \text { Other } & 2 \\ \text { Dindo grade } \geq \text { IIIb } & 1(0.8 \%) \\ \text { Suspected liver abscess } & 1\end{array}$

This article is protected by copyright. All rights reserved. 
Table 4. Logistic regression analysis local recurrence

$95 \%$ confidence

\begin{tabular}{llll} 
Factors & p-value & Odds ratio & interval \\
\hline Tumor $>3 \mathrm{~cm}$ & & & $.935-16.360$ \\
Child stage & .062 & 3.912 & $.439-4.321$ \\
BCLC stage & .582 & 1.378 & $.327-3.006$ \\
Previous HCC treatment & .997 & 1.002 & $.294-2.110$ \\
Close proximity (within $5 \mathrm{~mm}$ ) to major blood vessels $(>3 \mathrm{~mm})$ & .014 & .175 & $.044-.704$ \\
Tumor within 10mm of capsule & .637 & .789 & $.295-2.113$ \\
Distance to previous resection site/Clip <10mm & .741 & .717 & $.100-5.162$ \\
Number of tumors treated in 1 session & .115 & 1.271 & $.545-2.962$
\end{tabular}

This article is protected by copyright. All rights reserved. 


\section{Figure legends}

Figure 1. Work-Flow of SMWA showing (A) Planning phase: planning and simulation of the ablation probe trajectory. The target tumor (red circle) can be segmented automatically or manually, the expected ablation zone (green circle) is simulated according to the energy and time that will be applied. (B) Navigation phase: navigated positioning of ablation probe. The aiming device ensures stability and guides the insertion of the probe. (C) The computer calculates the required length of the inserted ablation probe. (D, E) Probe validation and ablation phase: validation of the intrahepatic ablation probe position followed by ablative treatment. If necessary the probe can be retracted or further inserted according to the calculations of the navigation system. (F) Ablation zone validation phase: overlay of pre- and post-ablation images in order to determine the safety margin and to plan another ablation if necessary.

Figure 2. (A) Distribution of treated lesions in the liver in relation to liver segments, (B) distribution of difficult-to-treat lesions in relation to their neighboring anatomic structures.

Figure 3. Kaplan-Meier curves showing local tumor progression-free survival (A) correlated with tumor size $\geq 3 \mathrm{~cm}$, and overall survival (B-D) correlated with (B) BCLC stage, (C) Child class and (D) previous HCC treatment.

Figure 4. (A) Explanted liver with several SMWA-treated lesions, one opened to demonstrate the necrosis, (B) HE-staining of ablation zone showing complete necrosis.

This article is protected by copyright. All rights reserved. 

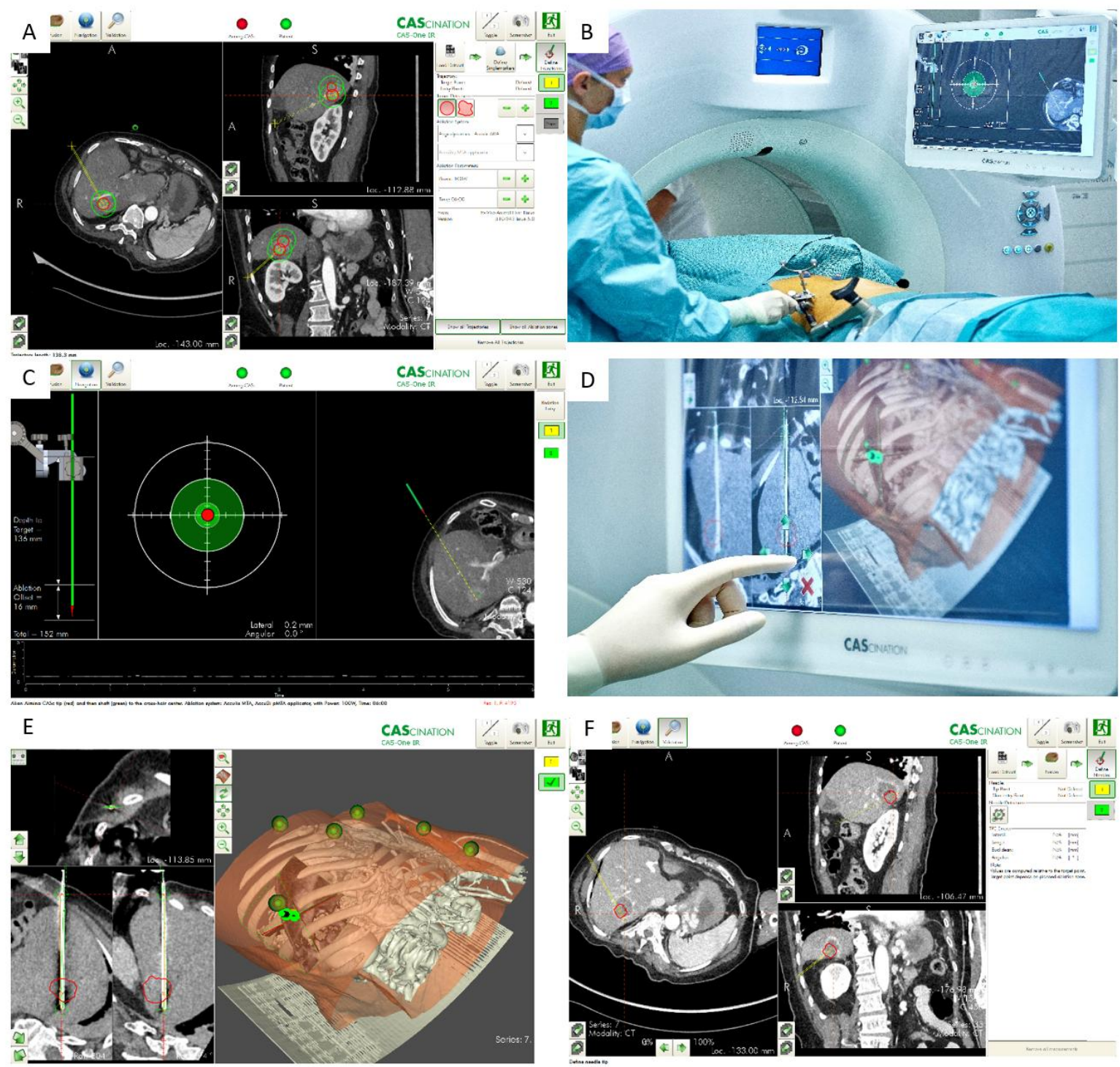

This article is protected by copyright. All rights reserved. 


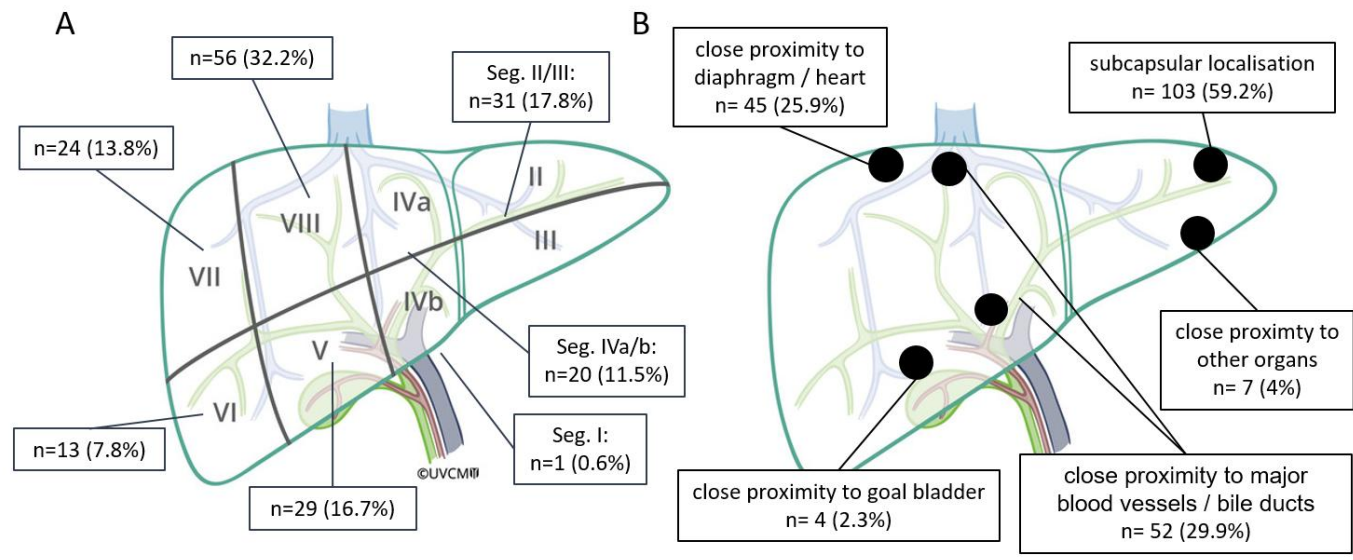

This article is protected by copyright. All rights reserved. 
A

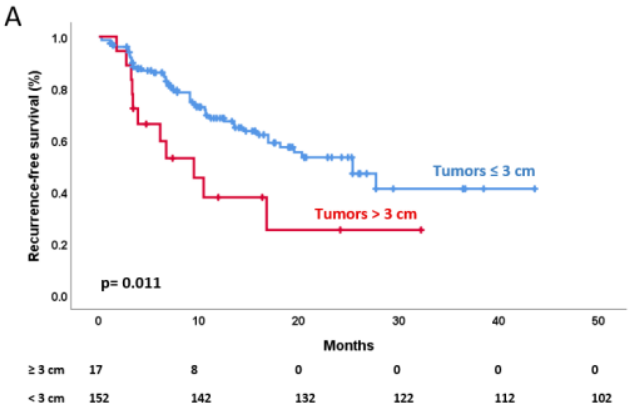

C

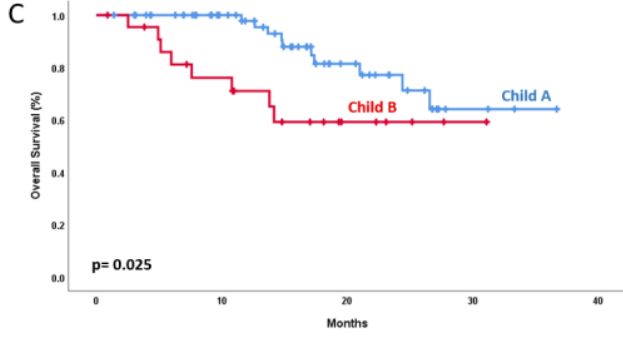

$\begin{array}{llllll}\text { Child class A } & 63 & 54 & 44 & 34 & 24\end{array}$
B

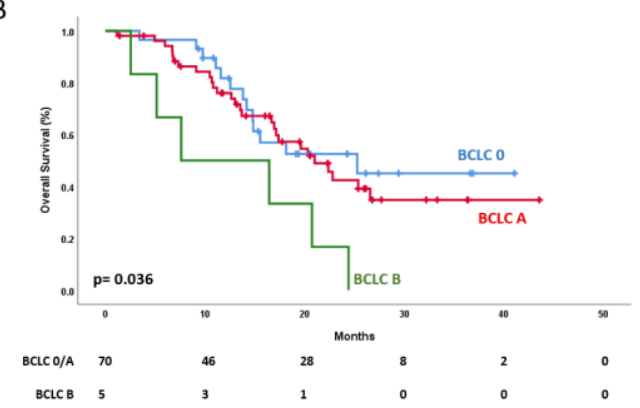

D

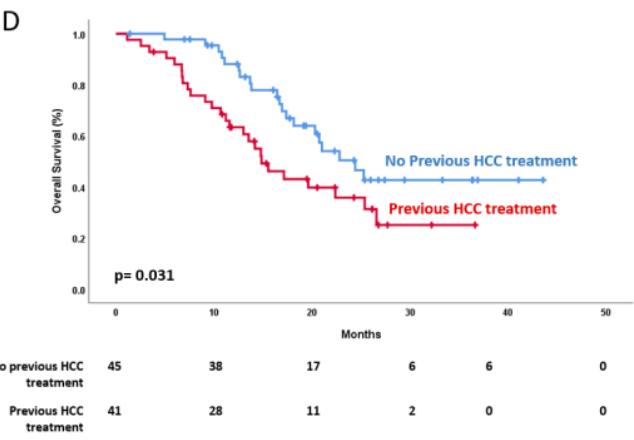

This article is protected by copyright. All rights reserved. 

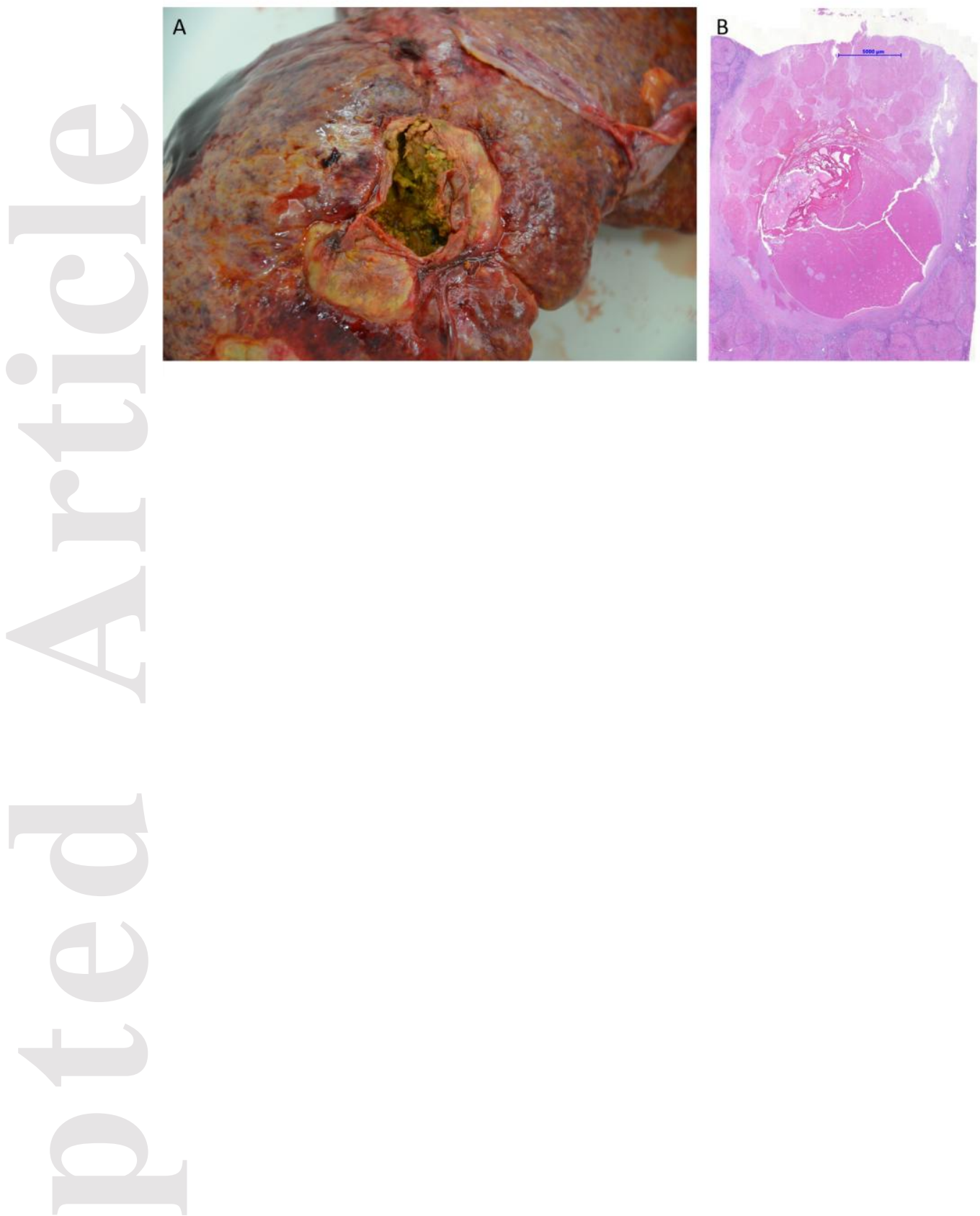

This article is protected by copyright. All rights reserved. 